\title{
Large-scale provision of frequency control via V2G: The Bornholm power system case
}

\author{
Zecchino, Antonio; Prostejovsky, Alexander Maria; Ziras, Charalampos; Marinelli, Mattia
}

Published in:

Electric Power Systems Research

Link to article, DOI:

10.1016/j.epsr.2018.12.027

Publication date:

2019

Document Version

Early version, also known as pre-print

Link back to DTU Orbit

Citation $(A P A)$ :

Zecchino, A., Prostejovsky, A. M., Ziras, C., \& Marinelli, M. (2019). Large-scale provision of frequency control via V2G: The Bornholm power system case. Electric Power Systems Research, 170, $25-34$.

https://doi.org/10.1016/j.epsr.2018.12.027

\section{General rights}

Copyright and moral rights for the publications made accessible in the public portal are retained by the authors and/or other copyright owners and it is a condition of accessing publications that users recognise and abide by the legal requirements associated with these rights.

- Users may download and print one copy of any publication from the public portal for the purpose of private study or research.

- You may not further distribute the material or use it for any profit-making activity or commercial gain

- You may freely distribute the URL identifying the publication in the public portal 


\title{
Large-scale Provision of Frequency Control via V2G: the Bornholm Power System Case
}

\author{
Antonio Zecchino, Alexander M. Prostejovsky*, Charalampos Ziras, Mattia Marinelli \\ Center for Electric Power and Energy, Technical University of Denmark, \\ DTU Risø Campus, Frederiksborgvej 399, 4000 Roskilde, Denmark
}

\begin{abstract}
This paper assesses the impact of electric vehicles (EVs) providing primary frequency regulation via vehicle-to-grid (V2G) technology. The aim of the work is to define a set of recommendations in order to guarantee a stable large-scale deployment of EV fleets as primary reserve providers. A realistic fleet model is proposed, which emulates the aggregated response of a number of EVs characterized by V2G hardware response times derived in laboratory tests. The effects of primary frequency control via EV fleets replacing conventional generating units are assessed with a sensitivity study in a single-bus power system with growing fleet sizes and response times. Two recommendations are derived to guarantee safe and stable operation: Recommendation 1 requires the share of EVs providing primary reserve to be smaller than the reserve from conventional units; Recommendation 2 requires response times below a given limit value, calculated as a function of the following power system parameters: the system inertia, the total primary reserve over the rotating generation capacity, and the employed droop gain. The full $60 \mathrm{kV}$ power system of the Danish island of Bornholm is then employed to evaluate the validity of the proposed requirements on a real system with complex dynamics, non-linearities and voltage dependencies.
\end{abstract}

Index Terms - electric vehicles; electric vehicles aggregation; frequency control; frequency stability.

\footnotetext{
*Corresponding author: tel: 0045 93511216, e-mail: alepros@elektro.dtu.dk
} 


\section{Introduction}

Electric vehicles (EVs) are considered promising sources of power system services, provided that their individual responses are properly aggregated in order to enable a safe and stable replacement of conventional sources. By modulating their battery charging/discharging process, EVs can perform vehicle-to-grid (V2G) services such as primary frequency control (PFC). The research emphasis in the field is put, among others, on EV fleet modelling for V2G services [1]-[3], combined smart charging and frequency regulation [4]-[6], and impact on the distribution level [7]-[9]. Studies on improvements in islanded power systems dynamics with high shares of renewable generations are also found in the literature [10]-[14]. For example in [10], [11], the frequency control actions implemented in EV controllers include an innovative inertial emulation logic, to counteract the reduction of system inertia due to large-scale generation from renewable energy resources. However, most of the contemporary literature rarely considers some technical hardware aspects are when modelling EVs for demand response purposes. As the primary function of an EV is transportation, their components are not designed to offer power system services, and thus many technological barriers need to be overcome when they are aggregated and controlled [15]. Critical response times of the aggregated EV fleet, as well as the need for each EV to comply with the ISO 15118 technical standard requirement of charging/discharging rate granularity, play an important role when dynamically assessing the response characteristics [16]. In fact, relatively large discrete step responses may trigger frequency stability problems, as presented in the literature within the domain of demand response [17]-[21], and also experienced in an experimental microgrid with smart-charging EVs [22], [23]. The stability of the power system may be jeopardized by V2G EV fleets in case of simultaneous and high ramping-rate responses, especially under large response delays. The state-of-the-art is lacking of exhaustive contributions on this topic: investigations are proposed only in [20] and in [21]. In Ref. [20] the authors propose a decentralized control scheme to assign randomized delay times on each individual $\mathrm{EV}$, which reacts by setting one of the three possible states (full charging, idle or full discharging) instead of considering a linear control modulation. In [21] EV droop 
controllers are designed for a centralized control scheme in a way to ensure the same stability margin with and without EVs performing PFC control. However, the typical delay accounting for the EV activation is not implemented.

In the present manuscript we aim at assessing the potential impact of aggregated $\pm 10 \mathrm{~kW}$ off-board EV chargers performing PFC on a real power system, relying on centralized control schemes already operating in field trial applications [24]. The choice of the $10 \mathrm{~kW}$ size is motivated by the outcome of the Danish founded demonstration projects Parker and ACES [25], [26], where the suitability of such chargers for the provision of grid services is recognized by the involved stakeholders. In particular, the development of commercial applications employing commercial fleets within companies or municipalities is of high interest, which enables high availability for reserve capacity during the evening time. Firstly, the employed EV fleet model is presented and characterized with realistic parameters obtained from commercial V2G hardware tests [27]. Secondly, the stability margin of the model is investigated, and the need for dedicated recommendations for grid operators is presented in terms of PFC from EVs replacing PFC provided by conventional generation units (CGUs).

The main contribution of this paper is the definition of two recommendations that EV fleet operators performing PFC need to fulfill in order to participate in the regulating market. In particular, limit values are found in terms of safe EV response times and overall primary reserve share from V2G units. This is obtained by carrying out a stability assessment, implementing accurate aggregated EV models and a representative single-bus power system testbed. The investigation is then extended to a real system with complex dynamics, non-linearities and voltage dependencies of the units, allowing the validation of the identified recommendations as well as the confirmation of the need for proper safety factors and simultaneous fulfilment of the two recommendations. The analysis is performed on the Danish island of Bornholm once it is disconnected from the mainland and therefore lacking of large synchronizing torques. The grid is therefore considered an excellent test case for investigating stability issues already arising with a limited number of 
chargers in case of slow time responses. The grid is implemented at 60 and $10 \mathrm{kV}$ levels with charging stations aggregated at the $10 \mathrm{kV}$ busbars. Frequency and voltage stability are investigated by means of RMS simulations in DIgSILENT PowerFactory software environment under different EV penetration scenarios as well as fleet response characteristics. The findings of this investigation will support system operators facing the future challenges due to frequency service procurement by EVs.

The paper is structured as follows. Section 2 presents the current framework for frequency control in the Nordic synchronous region and characterizes the power system of Bornholm Island. Section 3 describes the employed fleet model characterized with realistic parameters. Section 4 presents a single-bus case investigation, defining a set of recommendations in terms of critical activation times, and share of PFC from EVs for a given power system. In Section 5 the real power system of Bornholm is implemented in detail and realistic scenarios are investigated, and results are discussed. Conclusions are in Section 6.

\section{Conventional System Frequency Control}

This section first presents a summary of the current framework for frequency control in the Nordic synchronous region, and then it describes in detail the power system of the Danish island of Bornholm, which belongs to the Nordic area. The aim is to investigate barriers and opportunities for the provision of power system services via aggregated electric vehicles within the context of the Nordic frequency control framework, exploiting a testbed that can be operated also in islanded mode, i.e., when frequency control becomes more challenging due to less synchronous rotating mass.

\subsection{Framework for Frequency Control in the Nordic Area}

In general, frequency control is achieved in three subsequent phases, namely: (i) Primary frequency control, (ii) Secondary power-frequency control and (iii) Tertiary control [28]. The Regional Group Nordic (RGN) synchronous area is composed by the interconnected power systems of Norway, Sweden, Finland, and Eastern Denmark (DK2). In the RGN synchronous area, primary frequency control is achieved via two separate services: frequency-controlled normal operation reserve (FNR), activated linearly with no dead band 
for all frequency deviations within $\pm 100 \mathrm{mHz}$, and frequency-controlled disturbance reserve (FDR), activated in addition to FNR only when system frequency drops below $49.9 \mathrm{~Hz}$. In the current framework there is no automatic secondary frequency control in the RGN power system, whereas tertiary reserve is in place [29].

FNR is a symmetrical service, meaning that the same upwards and downwards reserve capacity must be provided. According to the service requirements, the reserve has to be provided within 150 seconds [29]. The minimum size of total FNR reserve that has always to be procured in the RGN is $600 \mathrm{MW}$, divided proportionally among transmission system operators (TSOs). FDR is a non-symmetrical service, as the involved units respond with only frequency up-regulation by linearly injecting power into the system when the measured frequency is below $49.9 \mathrm{~Hz}$, with full reserve activation at $49.5 \mathrm{~Hz}$. The $50 \%$ of the response has to take place within $5 \mathrm{~s}$, whereas the remaining $50 \%$ within an additional $25 \mathrm{~s}$.

The listed requirements set the basis for benchmarking the performance of the simulation activities performed on the real power system of the Danish island Bornholm, which is described in the following.

\subsection{Description of the Bornholm power system}

Bornholm is a Danish island in the Baltic Sea, located in the east of Denmark and the south of Sweden. The Bornholm electric power system is composed of distribution networks at three voltage levels: $60 \mathrm{kV}$, $10 \mathrm{kV}$ and $0.4 \mathrm{kV}$ [30], [31]. A $43.5 \mathrm{~km}$ long sea cable at $60 \mathrm{kV}$ with $60 \mathrm{MW}$ capacity connects the island to the Swedish system, which means that the Bornholm system is electrically coupled with the Nordic power system [32]. As from an electricity market and regulatory framework point of view the system belongs to the RGN, frequency control is performed as indicated in the previous subsection. Occasionally, the sea cable connection to Sweden is disrupted due to maintenance or incidents, forcing the Bornholm power system to run into islanding mode. During these periods, system frequency control is performed by the local distribution system operator (DSO) Bornholm Energi \& Forsyning (BEOF) relying on a set of units that provide conventional reserve, while at the same time shutting down most of the wind generators. As the goal 
of the proposed investigation is to replace conventional generation units employed for reserve with EV fleet providing frequency control via V2G, the islanded operation mode is studied.

The grid is modelled at the $60 \mathrm{kV}$ medium voltage (MV) level with real models of all the $60 \mathrm{kV}$ lines along with the $60 / 10 \mathrm{kV}$ substations. Aggregated loads, conventional generation units, renewable energy plants (wind turbines and photovoltaics (PV)) and EV fleet models are connected at the $10 \mathrm{kV}$ buses, and the detailed $10 \mathrm{kV}$ lines are not modelled since the $10 \mathrm{kV}$ system is not presenting any potential overloading issues. Since the aim of the analysis is the assessment of large-scale V2G employment on a system level, the complete $60 \mathrm{kV}$ grid is considered sufficient. The $60 \mathrm{kV}$ grid is shown in Fig. 1, with names and locations of the nodes with 60/10 kV substations. The $60 \mathrm{kV}$ network has 16 60/10 kV substations, $2360 / 10 \mathrm{kV}$ transformers with On-Load Tap Changers, and 22 cables and overhead lines of a total length of $131 \mathrm{~km}$. The peak load in Bornholm is $63 \mathrm{MW}$, whereas the minimum load is $13 \mathrm{MW}$. The complete generation set updated in May 2018 includes:

- 16 MW biomass combined heat and power plant (CHP) with steam turbine, named Blok 6. It has an inertia time constant $2 H=6.4 \mathrm{~s}$ and apparent power $S=46.8$ MVA. It is equipped with primary frequency droop control at $2 \%$, and automatic voltage regulator. The unit responds with a ramping rate of 0.2 $\mathrm{MW} / \mathrm{min}\left(1.25 \% \mathrm{P}_{\mathrm{nom}} / \mathrm{min}\right)$. As a side note, if it runs with coal/oil it can be boosted to 24/36 MW, respectively.

$-2 \cdot 1 \mathrm{MW}$ biogas CHP gas turbine, each with inertia $2 H=5.6 \mathrm{~s}$ and apparent power $S=1 \mathrm{MVA}$. These units are not equipped with primary frequency droop control.

- $37 \mathrm{MW}$ wind (24 machines <100kW; 16 machines between 100 and $1000 \mathrm{~kW} ; 17$ machines $>1000 \mathrm{~kW}$. The largest machines are three Siemens 2.3 MW wind turbines at the 60/10 kV substation in Hasle).

- 23 MW PV (8 MW distributed on rooftops at $0.4 \mathrm{kV} ; 2$ newly-installed $7.5 \mathrm{MW}$ PV plants at $10 \mathrm{kV}$ at the secondary sides of the $60 / 10 \mathrm{kV}$ substations in Aakirkeby and Bodilsker).

On top of the above listed generating units, there are other conventional units utilized only during islanded 
operation, for a total amount of $58 \mathrm{MW}$ of reserve. One of these conventional fossil fuel units utilized only for primary frequency reserve provision will then be replaced with a number of EV fleets in the following simulation studies. As of today, the total primary reserve portfolio includes:

- $25 \mathrm{MW}$ oil-powered steam turbine, named Blok 5. It has an inertia $2 H=8.6 \mathrm{~s}$ and an apparent power $S=29.4$ MVA. It is equipped with primary frequency droop control at $2 \%$, and an automatic voltage regulator. The unit responds with a ramping rate of $0.25 \mathrm{MW} / \mathrm{min}\left(=1 \% \mathrm{P}_{\text {nom }} / \mathrm{min}\right)$. It is important to note that the droop control of Blok 5 is generally not used in conjunction with Blok 6 due to hunting issues.

- 4.4.5 MW diesel generators, each equipped with 2\% frequency droop control and voltage control. Each unit has inertia $2 H=8 \mathrm{~s}$, whereas two units have $S=5.8$ MVA and the others $S=6.3$ MVA.

$-10 \cdot 1.5 \mathrm{MW}$ diesel generators, named Blok 7. Each unit has an inertia $2 H=1.1 \mathrm{~s}$ and an apparent power $S=2$ MVA. They are not equipped with primary frequency droop nor voltage control. Each unit responds with a ramping rate of $1 \mathrm{MW} / \mathrm{min}\left(66 \% \mathrm{P}_{\mathrm{nom}} / \mathrm{min}\right)$.

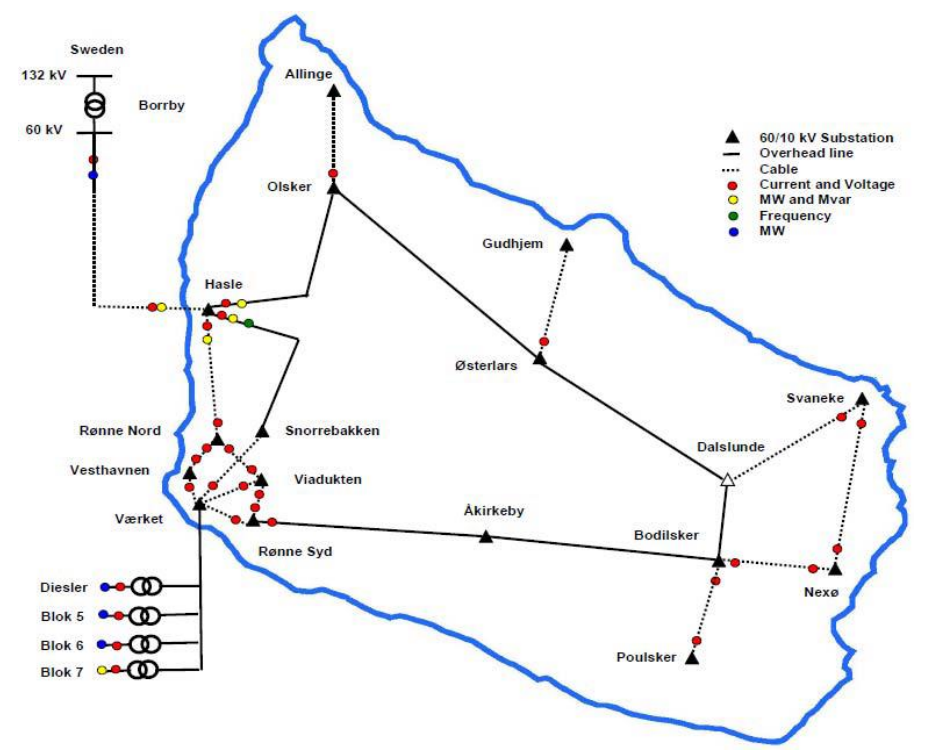

Fig. 1. Bornholm Island $60 \mathrm{kV}$ grid with major generation units and nodes with $60 / 10 \mathrm{kV}$ substations. 
Today on the island of the Bornholm there are more than 17000 internal combustion engine cars. The share of EVs will increase dramatically in the coming years, according to the Nordic EV Outlook 2018 report [33]. As it is expected that a number of EVs will constantly be available for V2G services, realistic models of controlled EV fleets need to be developed when assessing the major impacts on the power system. Already today, $2110-\mathrm{kW}$ chargers with bidirectional capability are employed to provide frequency regulation. In the next section we propose the novel EV fleet model utilized for power system studies.

\section{Aggregated EV fleet modelling}

In this section we present the adopted EV aggregation model, which is commonly utilized for power system studies. The fleet model is then characterized with real V2G hardware test results.

\subsection{Adopted EV Fleet Aggregation Model}

Given a population of $N E V$ chargers indexed by $i$, the most common representation of their response to a change in their power output is via a transfer function of the following form:

$$
H_{i}(s)=\frac{k_{E V, i}}{1+T_{E V, i} s} e^{-\tau_{i} s}
$$

where $k_{E V, i}$ is the controller's gain, $T_{E V, i}$ the first-order time constant, and $\tau_{i}$ the response delay.

The adopted aggregation model is a model where average values for the three sets of parameters are used, along with a gain $N$, representing the fleet size. A good approximation of the actual response of $N E V$ chargers is given by the transfer function $R_{E V}(s)$ :

$$
R_{E V}(s)=\frac{N \overline{k_{E V}}}{s+\overline{T_{E V}} s^{2}} e^{-\bar{\tau} s}
$$

The symbol $\overline{(\cdot)}$ denotes the average value of the three parameters in Eq. (1) (gains, first-order time constants and response delays) for the $N$ considered EVs. This model is named average model in the rest of the paper.

\subsection{Characterization with Real EV Response Times from Lab and Field Tests}

The presented average model is characterized with parameters derived from test results, and is adopted for the stability investigation when large-scale provision of frequency control is achieved via V2G technology. 
In this subsection, we present the main outcome of tests on real $\pm 10 \mathrm{~kW} \mathrm{V2G}$ chargers responding to charging/discharging control signals, setting power set-points both in a local and in a remote fashion, meaning that the control signal has been computed locally and remotely, respectively. This enabled us to derive the activation time of only the employed hardware, and to assess the additional communication latencies on the total activation time when an aggregator acts remotely. It is worth mentioning that the remote
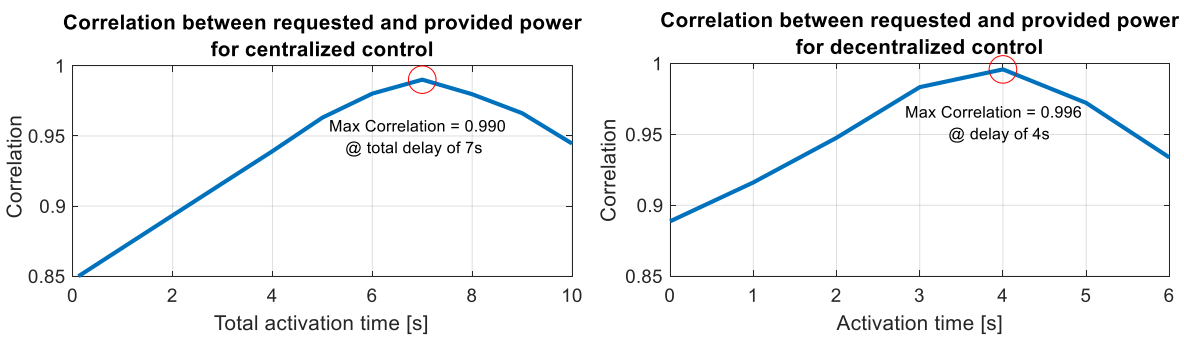

Fig. 2. Correlation between requested and provided power for remote and local control, which enabled us to derive the EV delays. control test setup includes the communication and control infrastructure utilized by an actual EV aggregator, operating in on-field projects such as the Danish-funded projects ACES [26] and Parker [25]. Appropriate test patterns with all the possible charging/discharging set-points were sent to the V2G chargers, as presented in the dynamic characterization tests presented in Ref. [27], allowing the calculation of the most probable response times of real commercial V2G-capable hardware.

The main test results are shown in Fig. 2, which reports the correlation between the requested and provided power when applying different time shifts to one of them for the whole duration of the test. This allows the estimation of the most probable activation time for both test setups, which resulted to be $7.0 \mathrm{~s}$ and $4.0 \mathrm{~s}$ for the remote and local control case, respectively.

One has to note that these results were obtained for one type of $\mathrm{V} 2 \mathrm{G}$ chargers and one type of control and communication infrastructure in case of remote control. Thus, slightly different results can be obtained in case of faster or slower hardware and/or communications. However, such results provide a valuable asset for the characterization of the proposed EV fleet model, as well as for the analysis of power system stability aspects related to frequency regulations via such resources. 


\section{Effects of Primary Frequency Control via EVs Replacing CGUs}

The purpose of this section is to provide general insights on the effects of large-scale PFC provision via aggregated EVs, and to outline safety recommendations to prevent system instability. Firstly, the dynamic performance of the aggregation models introduced in Section 3 is evaluated by investigating the frequency response of the models' transfer functions by their own as well as in a simulated simplified power system. Secondly, a set of simulations is carried out with increasing EV penetration share, for different activation times. The analysis proposes a method for defining critical activation times and V2G primary frequency regulation shares over the total primary reserve from conventional units. However, the numeric outcome of this investigation is not meant to be safely applicable in the real operation of any possible power system, but it should rather be considered as a benchmark for further grid analysis in more complete and complex simulation environments. In this context, we will implement the outlined recommendations in the detailed model of the Bornholm power system to evaluate their effectiveness in a real low-inertia system.

\subsection{Simplified Power System Layout and Modelling}

The first analysis is carried out by implementing the simplified power system in Fig. 3, with the single-bus layout proposed by [34] extended with the EV fleet models. The conventional generators are modelled with the transfer functions representation proposed in the literature, equipped with a proportional droop for primary frequency regulation. The system parameters are chosen in accordance with one possible islanded configuration of the Bornholm power system, and are listed in Table 1.

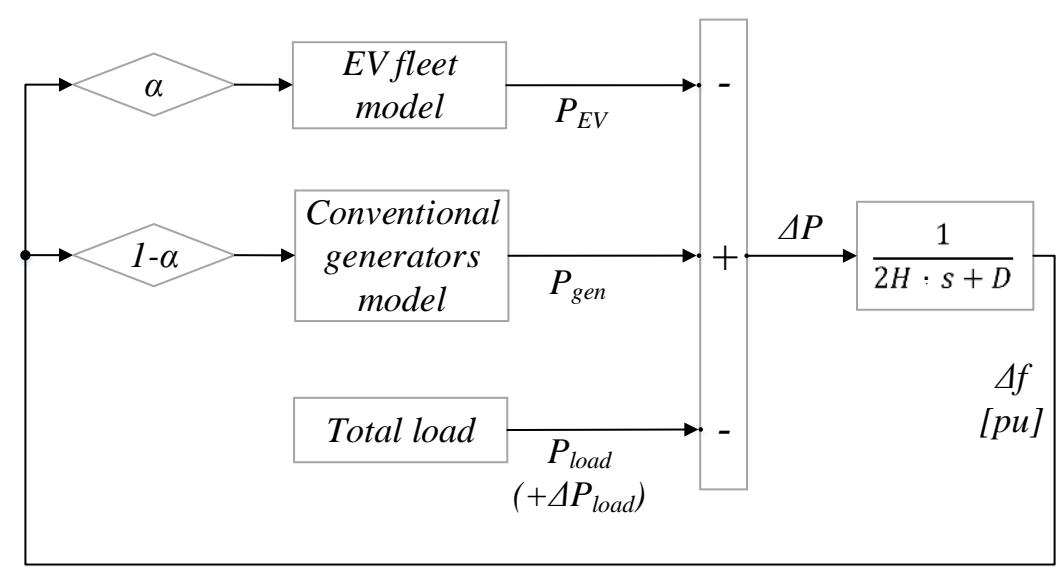

Fig. 3. Simplified power system with the classical single-bus layout. 
TABLE 1. SYSTEM PARAMETERS

\begin{tabular}{llll}
\hline \hline Parameter & Symbol & Value & Unit \\
\hline Base Frequency & $\omega_{0}$ & 314 & $\mathrm{rad} / \mathrm{s}$ \\
Inertia constant & $H$ & 3.6 & $\mathrm{~s}$ \\
Rated power & $S_{\text {rated }}$ & 108.2 & $\mathrm{MVA}$ \\
Damping factor & $D$ & 0 & $\%$ \\
Total load & $P_{\text {load }}$ & 60 & $\mathrm{MW}$ \\
Load step & $\Delta P_{\text {load }}$ & 2 & $\mathrm{MW}$ \\
Primary reserve & $P_{\text {reserve }}$ & 10 & $\mathrm{MW}$ \\
Primary frequency control normalized droop gain & droop & 2 & $\%$ \\
\hline \hline
\end{tabular}

The EV fleet is modelled as described in Section 3, acting with the same relative droop of the replaced CGU and with different participation factor $\alpha$ and activation times, which for the first set of simulation are considered to have an average value of $7 \mathrm{~s}$. The combined response of 100 individually simulated EVs with normally distributed delays and standard deviation of $0.1 \mathrm{~s}$ serves as the reference.

Frequency dynamics are modelled using the linearized swing equation $J \omega_{0} \dot{\omega}+D \omega=\Delta P_{\text {load }}$ and the inertia constant $H=\frac{J \omega_{s}{ }^{2}}{2 S_{\text {rated }}}$ according to [34]. No damping $(D=0)$ is considered as conservative assumption for the stability analysis. The Laplace-transformed representation of the grid is therefore

$$
G_{\text {grid }}(s)=\frac{f_{0}}{2 H S_{\text {rated }} s} .
$$

Despite the typical variety of types of rotating CGUs within the portfolio of an operating power system, in this preliminary study we consider only one type of CGU, i.e., diesel generator sets. In fact at this stage the aim is not a detailed power system analysis, but rather the provision of general insights on technical barriers of EV fleets management on a system level. The detailed power system with complete generation portfolio, real line and load models and voltage dependencies will be implemented later on, in order to validate the outcome of this first part of the study. The standard diesel model given in [35] is implemented, equipped with an electric control box:

$$
R_{C G U}(s)=\frac{1}{1+T_{g} S} \frac{1+T_{3} s}{1+T_{1} s+T_{1} T_{2} s^{2}} \frac{k_{C G U}}{1+T_{4} s} .
$$

The implemented EV aggregation model is the average model described by Equation (2), which guarantees a finer representation of a large-scale EV fleet with EV delays compared to the commonly used 
averaging models. The resulting dynamic system in open- and closed-looped form is so described by $L(s)$ and $T(s)$

$$
L(s)=G_{\text {grid }}(s)\left(R_{C G U}(s)+R_{E V}(s)\right), \quad T(s)=\frac{G_{\text {grid }}(s)}{1+L(s)}
$$

where $R_{E V}(s)$ is the average EV aggregation model. The share of EVs and conventional resources is expressed over the factor $\alpha$ as in

$$
k_{E V}=\alpha k, \quad k_{C G U}=(1-\alpha) k, \quad k=\frac{P_{\text {reserve }}}{f_{0}} \frac{1}{d r o o p}
$$

where $k$ is the absolute primary droop gain, and $P_{\text {reserve }}$ the total power allocated for primary reserve.

\subsection{Model Dynamics}

Fig. 4-a shows the Bode magnitude plot of the real EV fleet (used as reference) and the average model, where the input is the frequency deviation $\Delta f=f-f_{0}$ and the output is the power delivered by the EVs. As can be observed, magnitude and phase of the average model match well with the real EV fleet in the frequency range below $1 \mathrm{~Hz}$. At $1 \mathrm{~Hz}$ the deviation amounts to about $1 \mathrm{~dB}$, whereas a deviation of $3 \mathrm{~dB}$ is found for a frequency of $1.5 \mathrm{~Hz}$.

Within the given Bornholm power system context, we expect similar behavior of the models due to the smoothing effect of the grid's inertia and the $50 \%$ conventional resources, being $\alpha=0.5$ for this first simulation. This is confirmed in Fig. 4-b on the open-loop $L(s)$ of (5), where the characteristic system behaviors happen in frequency ranges a full magnitude below those seen in Fig. 4-a. Here, load power is the input signal and requested EV power the output. The results of the full EV fleet and the aggregation model

(a)

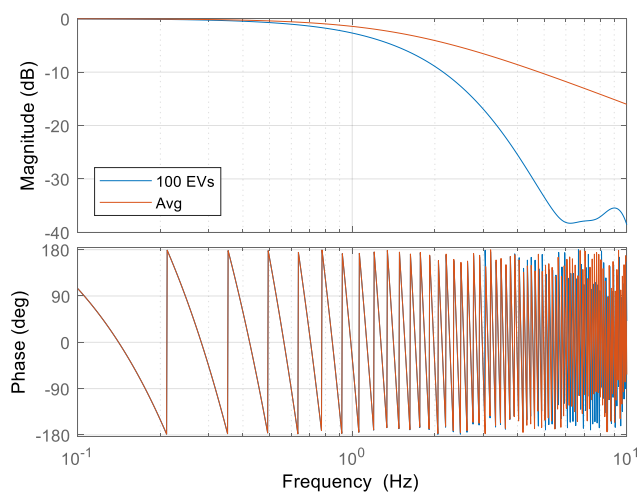

(b)

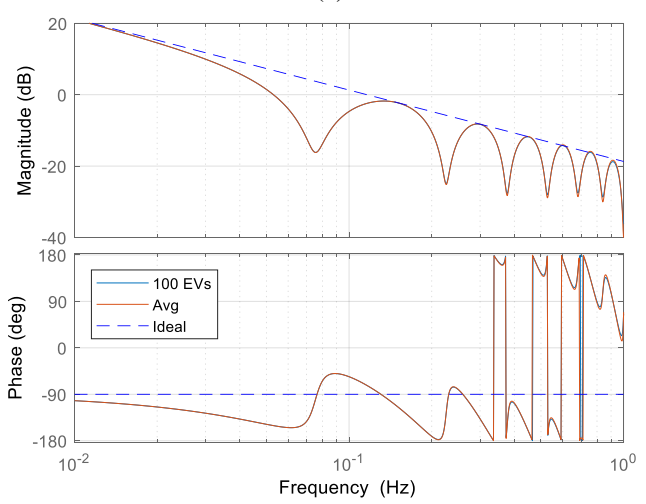

Fig. 4. Bode plot of the EV fleet and the corresponding aggregated average model in its open-loop form (a) and within a simulated one-bus system (b) Input is the frequency deviation $\Delta f$, output the delivered $\mathrm{EV}$ power normalized to their nominal droop gain. The phase is wrapped between \pm 180 degrees. 
are furthermore compared to the response of ideal conventional primary resources with no dynamics of their own, shown by the dotted line in Fig. 4-b. The average model performs almost identical to the simulated fleet, rendering it valid for subsequent investigations.

\subsection{Stability Investigation}

In order to make more generalized statements on stability of primary support using EVs, the impact of the EV share $\alpha$ to the total primary reserve is now investigated. The linearized dynamic system can be written as

$$
\dot{x}(t)=A_{0} x(t)+A_{1} x(t-\tau), \quad \tau \geq 0
$$

with the $n$ states $x \in \mathbb{R}^{n \times 1}$, the system matrices $A_{0}, A_{1} \in \mathbb{R}^{n \times n}$ on which the normal and delayed states act, and the delay time $\tau$. Rearranging the strictly proper transfer functions (2)-(4) into the monic form $G(s)=\frac{b_{0}+b_{1} s+\cdots+b_{n-1} s^{n-1}+b_{n} s^{n}}{a_{0}+a_{1} s+\cdots+a_{n-1} s^{n-1}+a_{n} s^{n}}$ allows their transformation into the canonical state-space observer representation $A_{\text {grid }}=0, \boldsymbol{b}_{\text {grid }}=b_{\text {grid, }} A_{C G U} \in \mathbb{R}^{4 \times 4}, \boldsymbol{b}_{C G U} \in \mathbb{R}^{4 \times 1}$ and $A_{E V}=a_{E V}, \boldsymbol{b}_{E V}=b_{E V}$. By choosing

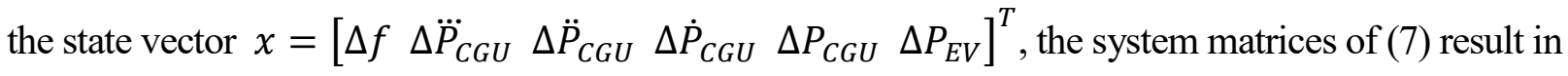

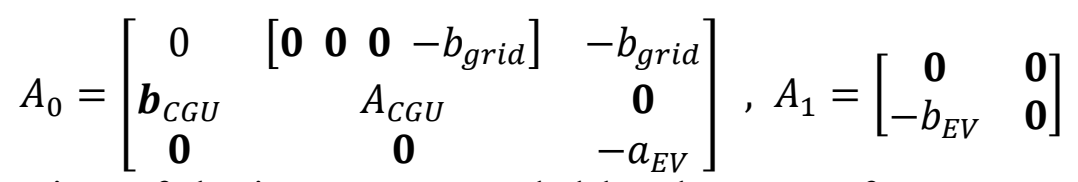

Using these system matrices of the instantaneous and delayed states, a frequency sweeping test as described by Theorem 2.1 in [36] is utilized, which allows to find the maximum share of EVs for which the system remains stable independently of the delay. Independence of delay is imperative, as the response of real EVs is non-deterministic and subject to various uncertain factors (battery management, communication systems, charging station electronics, etc.). The three necessary and sufficient conditions of the test are:

1) $A_{0}$ is stable (for $\tau \rightarrow \infty$ );

2) $A_{0}+A_{1}$ is stable (for $\tau \rightarrow 0$ );

3) $\rho\left(\left(j \omega I-A_{0}\right)^{-1} A_{1}\right)<1, \quad \forall \omega>0$, 
(a)

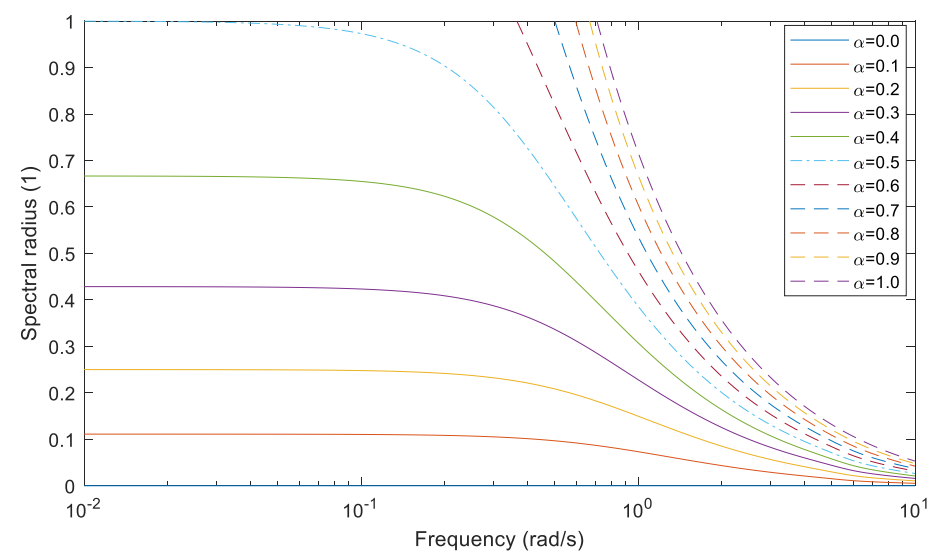

(b)

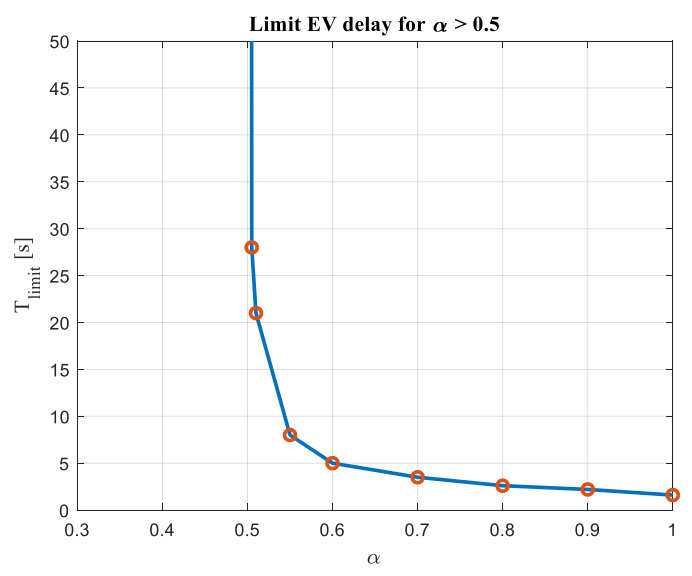

Fig. 5. Frequency sweep of the spectral radius as part of the frequency sweep test (a). (b) shows the Limit EV delay $T_{\text {limit }}$ for $\alpha>0.5$. A clear delay-independency of the stability is graphically confirmed for $\alpha<0.5$.

with $\rho(\cdot)$ as the spectral radius of a matrix. Conditions 1 and 2 are fulfilled for the given system because the corresponding eigenvalues are in the left half of the complex plane. Condition 3 is evaluated in Fig. 5-a, where we find valid solutions for $\alpha<0.5$. The system is stable independently of the delayed EV response for EV shares $<50 \%$. It is noted that the results mark the fundamental stability limit of the linearized system. Non-linearities inherent to real systems as well as voltage-related dynamics will generally decrease the available margin. For practical applications with the given EV/Diesel primary reserve mix, it is therefore recommended that the share of EVs stays below 50\% (Recommendation 1) in order to guarantee stable, EV delay-independent grid operation. For the sake of completeness, Fig. 5-b confirms that only for $\alpha>0.5$ the stable operation is limited by the critical time delays, which are inversely proportional to the EV share.

In order to take into account the probable reduction of the $\alpha$ limit in case of non-linearities and voltage-dependencies related to more complex systems, a recommendation in terms of maximum time delay is also introduced. In this respect, we hereby propose results from a sensitivity study that allowed the definition of a set of first-order equations, to calculate the maximum acceptable EV delay. It is important to note that the time limits are calculated for a share that is larger than the limit $(\alpha=0.55)$, in order to have delay-dependency of the stability margin. The analysis has been performed for different system parameters that could influence the results: the system inertia $2 H$, the primary reserve droop, and the index $\xi$, which 
gives an idea of the amount of total primary reserve over the rotating energy $E_{\text {rotating }}$ for the $n$ rotating CGUs with installed capacity $P_{n, i}$ that are connected to the system, as defined in Equation (9):

$$
\xi=\frac{P_{\text {reserve }}}{E_{\text {rotating }}} \quad, \quad E_{\text {rotating }}=\sum_{i=1}^{n} P_{n, i} 2 H_{i} .
$$

The influence of the three parameters on the critical EV delay as been assessed for a set of values: $2 H=$ $\{2.4,3.6,7.2,14.4\}$, droop $=\{\mathbf{0 . 0 2}, 0.03,0.04,0.05,0.06\}, \xi=\{0.01,0.02, \mathbf{0 . 0 3}, 0.04\}$. The dependency of the time limits on the three parameters is considered almost linear, as deducible also by the example in Fig. 6. The figure shows the dependency on the three parameters and the linear interpolation, performed to derive the three first order equations. So, the outcome of the proposed parametric study allows the identification of the coefficients $a_{i}$ and $b_{i}$ for the calculation of the time limit $T_{\text {limit, }}$ given the considered system parameters.

$$
T_{\text {limit }}= \begin{cases}a_{k} \cdot \text { droop }+b_{k} & \text { with } a_{k}, b_{k} \text { function of }(2 H, \xi) \\ a_{\text {iner }} \cdot 2 H+b_{\text {iner }} & \text { with } a_{\text {iner }}, b_{\text {iner }} \text { function of }(d r o o p, \xi) \\ a_{\xi} \cdot \xi+b_{\xi} & \text { with } a_{\xi}, b_{\xi} \text { function of }(\text { droop }, 2 H)\end{cases}
$$

The resulting coefficients are reported in Tables 2-4, which allow the calculation of the maximum response time of the EVs in order to prevent system instability. Note that the bold values both in the text and in the tables relate to the realistic islanded operation mode of the Bornholm power system, which gives a delay limit of $8 \mathrm{~s}$. So, we hereby deduct the second recommendation (Recommendation 2) for a safe and stable primary reserve provision from a fleet of EVs: $\boldsymbol{\tau}<\boldsymbol{T}_{\text {limii }} \boldsymbol{2}$, i.e., operate with a delay smaller than the half of the calculated $T_{\text {limit. }} 2$ is a safety factor, introduced to prevent operating too close to the limit and to take into account possible imperfections in the calculation of $T_{\text {limit }}$ given the extrapolation of the coefficients. 

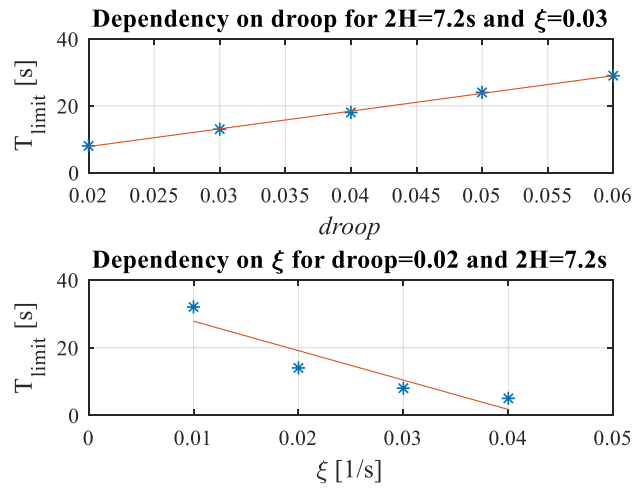

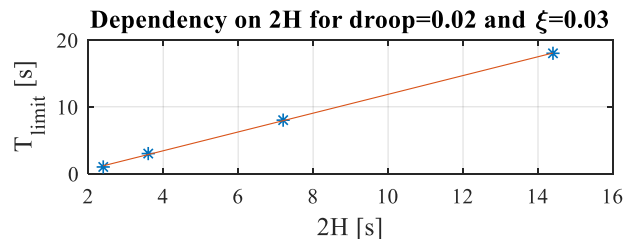

$2 \mathrm{H}[\mathrm{s}]$

Fig. 6. Dependency of the limit time $T_{\text {limit }}$ for different system parameters. It can be noticed that the approximation to a first-order equation for the dependency of the three parameters causes a relatively small error in the calculation of $T_{\text {limit }}$.

TABLE 2. COEFFICIENTS TO CALCULATE $T_{\text {LIMIT }}$ AS FUNCTION OF droop

\begin{tabular}{c|c|cccc}
\hline \hline \multicolumn{2}{c|}{} & \multicolumn{4}{c}{$2 \boldsymbol{H}[\mathbf{s}]$} \\
\cline { 3 - 6 } \multicolumn{2}{c|}{} & 2.4 & 3.6 & 7.2 & 14.4 \\
\hline \multirow{3}{*}{$\xi\left[\mathbf{s}^{-1}\right]$} & 0.01 & $\mathrm{a}=570 ; \mathrm{b}=-2.2$ & $\mathrm{a}=860 ; \mathrm{b}=-2.2$ & $\mathrm{a}=1720 ; \mathrm{b}=-3$ & $\mathrm{a}=3360 ; \mathrm{b}=-2.4$ \\
& 0.02 & $\mathrm{a}=270 ; \mathrm{b}=-2.4$ & $\mathrm{a}=400 ; \mathrm{b}=-2$ & $\mathrm{a}=800 ; \mathrm{b}=-2$ & $\mathrm{a}=1710 ; \mathrm{b}=-5.4$ \\
& 0.03 & $\mathrm{a}=170 ; \mathrm{b}=-2.4$ & $\mathrm{a}=250 ; \mathrm{b}=-2.2$ & $\mathbf{a}=\mathbf{5 3 0} ; \mathbf{b}=\mathbf{- 2 . 8}$ & $\mathrm{a}=1000 ; \mathrm{b}=-2$ \\
& 0.04 & $\mathrm{a}=111 ; \mathrm{b}=-2.12$ & $\mathrm{a}=164 ; \mathrm{b}=-1.9$ & $\mathrm{a}=360 ; \mathrm{b}=-2.4$ & $\mathrm{a}=720 ; \mathrm{b}=-2.8$ \\
\hline \hline
\end{tabular}

TABLE 3. COEFFICIENTS TO CALCULATE $T_{\text {LIMIT }}$ AS FUNCTION OF THE SYSTEM INERTIA $2 H$

\begin{tabular}{|c|c|c|c|c|c|}
\hline & \multicolumn{4}{|c|}{ droop } \\
\hline & & 0.02 & 0.03 & 0.04 & 0.05 \\
\hline \multirow{4}{*}{$\xi\left[\mathbf{s}^{-1}\right]$} & 0.01 & $\mathrm{a}=4.74 ; \mathrm{b}=-2.20$ & $\mathrm{a}=6.89 ; \mathrm{b}=-1.29$ & $a=9.08 ; b=-0.66$ & $\mathrm{a}=11.67 ; \mathrm{b}=-1.55 \mathrm{a}=13.96 ; \mathrm{b}=-0.81$ \\
\hline & 0.02 & $\mathrm{a}=2.24 ; \mathrm{b}=-2.20$ & $a=3.25 ; b=-1.66$ & $a=4.48 ; b=-2.40$ & $a=5.74 ; b=-2.88 \quad a=7.01 ; b=-3.37$ \\
\hline & 0.03 & $a=1.41 ; b=-2.20$ & $a=2.10 ; b=-2.26$ & $a=2.81 ; b=-2.40$ & $\mathrm{a}=3.31 ; \mathrm{b}=-2.23 \quad \mathrm{a}=4.17 ; \mathrm{b}=-1.77$ \\
\hline & 0.04 & $\mathrm{a}=0.98 ; \mathrm{b}=-2.12$ & $a=1.39 ; b=-2.02$ & $a=1.98 ; b=-2.40$ & $a=2.59 ; b=-3.12 \quad a=2.94 ; b=-2.26$ \\
\hline
\end{tabular}

TABLE 4. COEFFICIENTS TO CALCULATE $T_{\text {LIMIT }}$ AS FUNCTION OF $\xi$

\begin{tabular}{|c|c|c|c|c|c|c|}
\hline & & \multicolumn{5}{|c|}{ droop } \\
\hline & & 0.02 & 0.03 & 0.04 & 0.05 & 0.06 \\
\hline \multirow{4}{*}{$2 \mathrm{H}[\mathrm{s}]$} & 2.4 & $a=-281 ; b=10.35$ & $a=-441 ; b=17.35$ & $a=-610 ; b=24$ & $a=-740 ; b=30$ & $a=870 ; b=36.5$ \\
\hline & 3.6 & $\mathrm{a}=-441 ; \mathrm{b}=17.35$ & $a=-680 ; b=27.5$ & $a=-870 ; b=36.5$ & $a=-1100 ; b=46$ & $a=-1350 ; b=57$ \\
\hline & 7.2 & $a=-870 ; b=36.5$ & $a=-1290 ; b=55$ & $a=-1710 ; b=74$ & $a=-2180 ; b=95$ & $a=-2600 ; b=113.5$ \\
\hline & 14.4 & $a=-1740 ; b=75$ & $a=-2570 ; b=111.5$ & $a=-3360 ; b=148$ & $a=-4280 ; b=189$ & $a=-5200 ; b=229$ \\
\hline
\end{tabular}

\section{Validation on the real Bornholm (DK) Power System}

This section presents the validation study carried out on a simulation basis on the Bornholm system. The grid layout along with the load and generation portfolio during islanded operation is presented, and scenarios with a 2040 EV penetration are outlined. Aggregated EVs are modelled according to the average model, and the recommendations for preventing instabilities found in Section 4 are implemented. These analyses complete the study, assessing the applicability on a real and complex power system of the recommendations. 


\subsection{Definition of Scenarios}

The investigation is carried out on an islanded configuration of the Bornholm power system, in a probable 2040 scenario with $50 \%$ EV penetration, meaning that out of the total 17000 cars on the island, 8500 will be EVs [37]. We consider an evening hour (e.g., between 18:00 and 19:00) when we can realistically assume that $40 \%$ of the EVs are charging at home on the $3.7 \mathrm{~kW}$ slow charging mode (Mode 2), leading to about 12 MW of total extra load. This is added to the rather high evening load condition assumed to be 48 MW, leading to a total load of $60 \mathrm{MW}$. Furthermore we assume that a portion of the remaining EVs not charging at home are connected to V2G chargers and are available for grid frequency regulation. Specifically, the V2G-ready EVs could be the 5\% of the total EVs, i.e., 450 EVs: considering each vehicle interfaced with a $10 \mathrm{~kW}$ bidirectional charger, the total regulation capacity is equal to $\pm 4.5 \mathrm{MW}$. To make the analysis more realistic the fleet is not considered connected to a single bus of the grid, instead the $450 \mathrm{EVs}$ are connected to the four largest urban areas in the island with the following criteria: $225 \mathrm{EVs}$ are in the capital city of Rønne (EV fleet \#1), whereas the remaining $225 \mathrm{EVs}$ are equally split over the cities of Hasle, Nexø, and Svaneke, leading to an amount of 75 EVs per city $($ EV fleet \#2, \#3, \#4).

As for the generation portfolio, despite the today operation policy of disconnecting all renewable generation when the systems becomes islanded, we consider that a very high share of renewables is present. In particular, half of the generation (30 MW) coming from wind turbines, whereas no PV production due to the assumption of operating in evening time. The other half of the generation is coming from the two biogas plants (1 MW each), the CHP plant Blok 6 (operating at $8 \mathrm{MW} \mathrm{-} \mathrm{50 \%} \mathrm{of} \mathrm{full} \mathrm{power),} \mathrm{and} \mathrm{the} \mathrm{oil-powered}$ steam turbine Blok 5 (operating at $20 \mathrm{MW}-80 \%$ of full power). Furthermore one $4.5 \mathrm{MW}$ diesel unit is considered connected but operating at zero set-point, ready to react in case of frequency disturbances as primary frequency regulator. As the framework of the proposed validation simulation study is the real operating condition during an islanded configuration, some of the CGUs today employed only as back-up

units for primary reserve are included. In this configuration the system has a primary frequency control 
reserve capacity of $5 \mathrm{MW}$ over $200 \mathrm{mHz}$ from the Blok 5, and additional 4.5 MW which are available either by a dedicated 4.5 MW diesel unit (operating at zero set-point but connected as mere frequency regulation unit upwards), or alternatively by the V2G-capable EV fleet. This means that for the proposed study case the share of EVs participating in the reserve is $\alpha=0.45$, fulfilling Recommendation 1 presented in the previous section. Both the synchronous units and the V2G EV fleet operate with a relative droop of $2 \%$. In this islanded configuration the system inertia $H$ will be $3.63 \mathrm{~s}$ if the diesel is connected, and $3.60 \mathrm{~s}$ in case it is not connected. The destabilizing contingency is the loss of a $2 \mathrm{MW}$ wind turbine.

\subsection{Results}

Fig. 7 shows the effects of EVs replacing the diesel generator with $\alpha=0.45$ (fulfilling Recommendation 1) in case of different EV delays, with delays normally distributed around 1, 4, 7 and $10 \mathrm{~s}$ with standard deviation $\sigma=0.1$. In the Section 4 it was found that for this setup a response equal to or faster than $4 \mathrm{~s}$ is needed to fulfill Recommendation 2. This is guaranteed in the cases of $1 \mathrm{~s}$ and $4 \mathrm{~s}$ delay. The $7 \mathrm{~s}$ case would fulfill the recommendation only if the safety factor 2 is not be included, whereas the $10 \mathrm{~s}$ case is above the requirement.

With a very fast EV response (e.g., $1 \mathrm{~s}$ ) the fleet can perform well, as the frequency settles to the steady-state value $f_{\text {steady-state }}=49.65 \mathrm{~Hz}$ even faster than in the case of the diesel. In case of larger delays, frequency stability is compromised: with the EVs responding in $4 \mathrm{~s}$ and $7 \mathrm{~s}$, damped oscillations appear, with settling time that increases dramatically in the case of $7 \mathrm{~s}$, which is very close to the limit of $8 \mathrm{~s}$ found in Section 4 . It can be noticed that the fulfilment of Recommendation 2 including the safety factor 2 guarantees the frequency to settle much faster than in the case of $7 \mathrm{~s}$, justifying the need for the inclusion of the safety factor for safer operations. In case of $10 \mathrm{~s}$ delay, the frequency is not damped and stability is lost. At this point it is relevant to highlight the fact that, despite in a simplified system the share $\alpha=0.5$ would allow any possible EV delay without incurring in instabilities, here the complex dynamics that describe the real power system model's behavior are reducing the stability limits as instability conditions are found for $\alpha=0.45$ for a $10 \mathrm{~s}$ delay. This is due to the fact the implemented Bornholm power system now includes the different dynamics of the CGUs of 
the complete generation portfolio along with the models of lines, transformers and loads. This brings along correspondent non-linearities and voltage dependencies that could not be included in the preliminary analysis of Section 4, where a simplified single-bus power system was modelled. This shows the need for the additional requirement in terms of maximum EV time delay (Recommendation 2), as the limit of $\alpha=0.5$ may not be sufficient to guarantee an EV delay-independent system stability in such large and complex systems. This confirms the considerations presented in the previous Section, when it comes to cautionary recommendations, and the inclusion of Recommendation 2, which in addition to Recommendation 1 allows the definition of safe operative conditions with large-scale frequency control via EV fleets replacing CGUs.
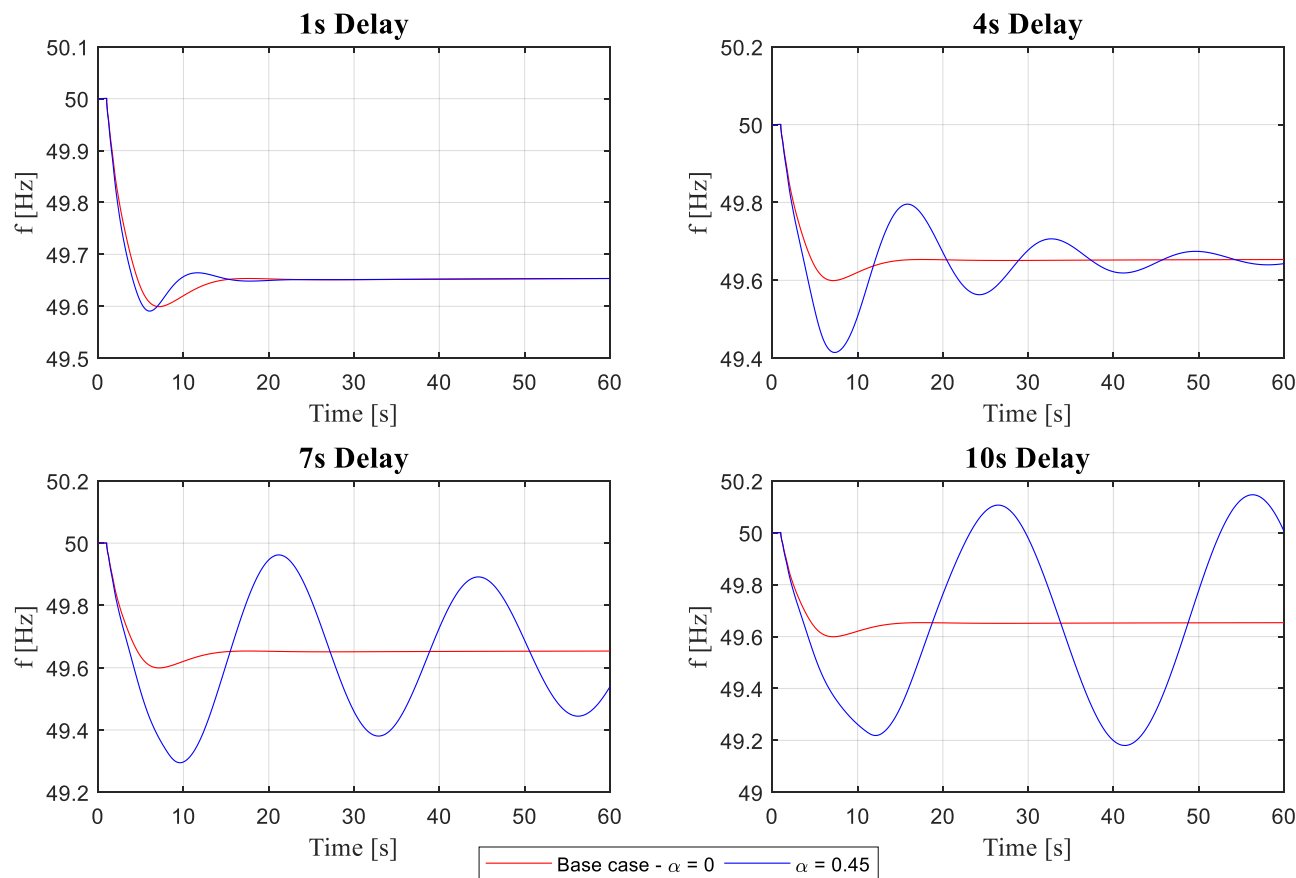

Fig. 7. Power system frequency behavior for $\alpha=0$ and $\alpha=0.45$ with increasing EV response times.

The voltage profiles at the connection buses for the different study cases as well as the power exchanges from EV fleets and diesel are reported in Fig. 8 and 9, respectively. It can be noticed that acceptable voltage levels are found at the $4 \mathrm{EV}$ fleets connection buses, as the RMS values of the line-to-ground bus voltages do not exceed the $10 \%$ of deviation from the nominal value, as required by the European grid standard EN 50160. As for the provided power, for the $\alpha=0.45$ cases at steady-state the sum of the powers from the EV fleets corresponds to the reserve that is provided by the diesel unit in the base case scenario with $\alpha=0$. The power 
provided from the EV fleets has negative sign, since they are modelled with the load convention. Moreover, as expected, it can be noticed that fleet \#1 provides triple the power of fleets \#2, \#3 and \#4, being the fleet sizes $225,75,75$ and $75 \mathrm{EVs}$, respectively.
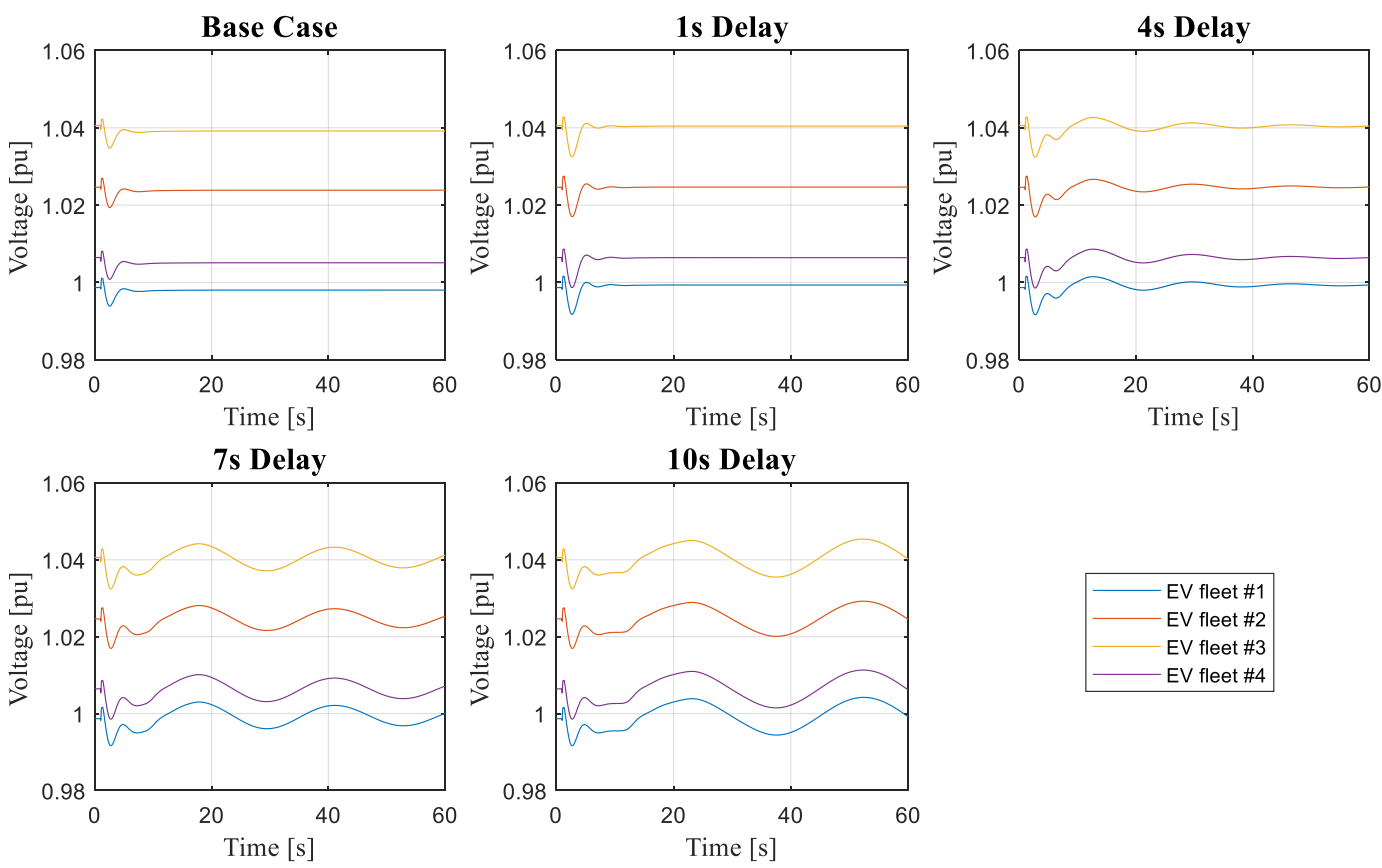

Fig. 8. Line-to-ground voltages at the EV fleet buses.
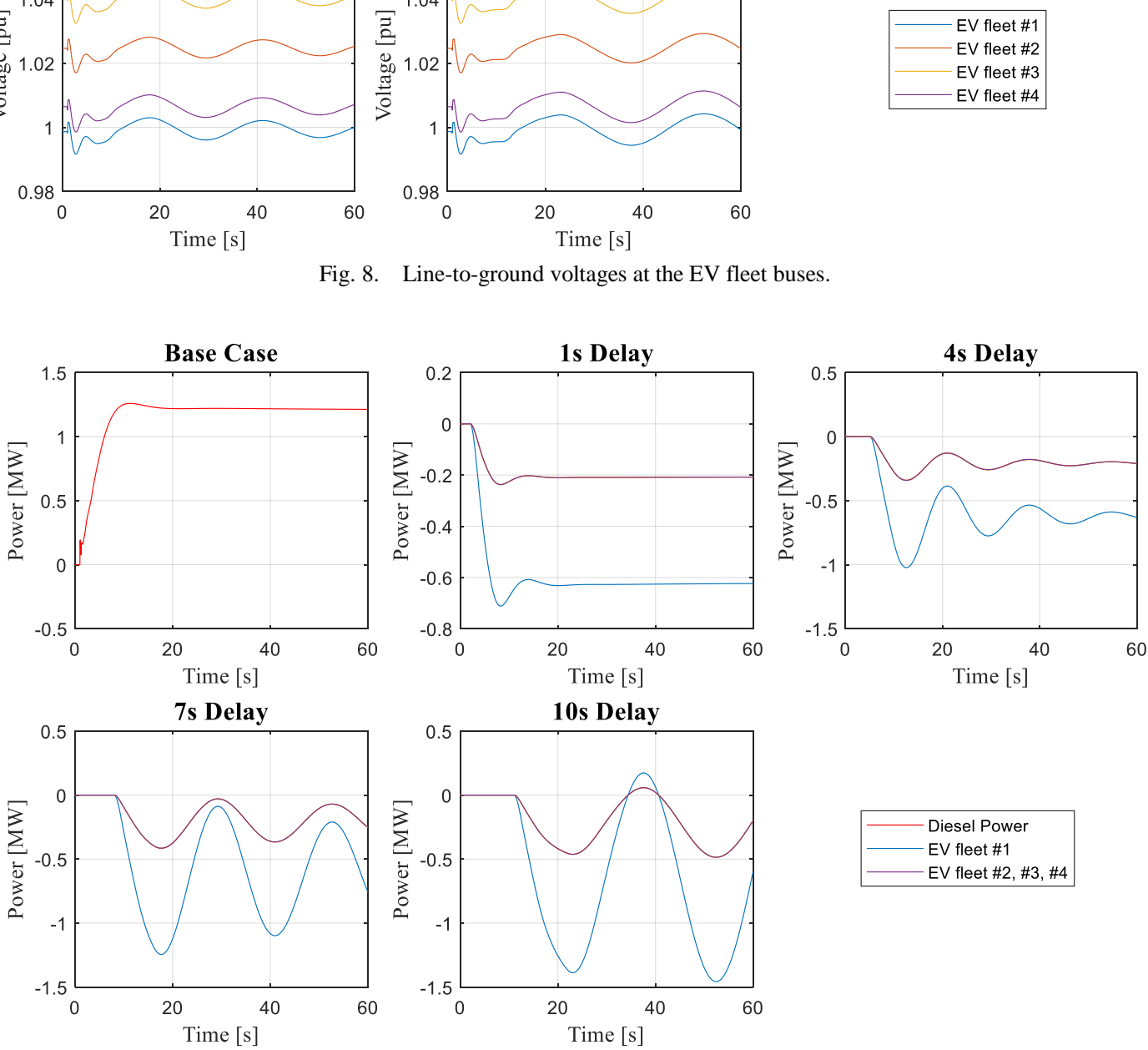

Fig. 9. Power profiles of the Diesel generator in case of $\alpha=0$, and of the $4 \mathrm{EV}$ fleets in the cases of $\alpha=0.45$. 


\section{Discussion and Conclusions}

This work investigated the impact of EV fleets providing primary frequency regulation via V2G technology as well as the importance of introducing requirements for large-scale applications. The aim of the paper was to assess the implications of large-scale employment of EV fleets as primary reserve providers given growing activation delays and shares of primary reserve acquired from non-conventional rotating units. An EV fleet model was proposed to emulate the aggregated response of a number of EVs reacting with realistic V2G hardware response times, obtained through laboratory tests. The stability limits when operating a representative power system are investigated to assess the effects of primary frequency control via V2G-capable EV fleets replacing conventional generation units.

The first part of the analysis aimed at defining general recommendations in terms of response time and size of the overall fleet, given the implemented simplified single-bus power system. Two recommendations with conservativeness considerations are derived to guarantee safe and stable operation. Specifically, Recommendation 1 requires to operate with a share of primary reserve from EVs that would not exceed the reserve from conventional rotating units $(\alpha<0.5)$. Recommendation 2 requires response times below the half of a limit value $T_{\text {limit }}$ that can be calculated as function of the system inertia, of the total primary reserve over the rotating generation capacity, and of the employed droop gain.

The second part of the study proposes a set of simulations of an islanded configuration with $50 \%$ of renewables of the Bornholm power system, with the scope of evaluating the reliability of the proposed recommendations in a system with complex dynamics, non-linearities and voltage dependencies of the units. Results show that only one recommendation was not sufficient: for $\alpha=0.45$ instability may occur for relatively large EV response times, despite the share below the 0.5 limit. In fact, although the 0.5 limit was valid in the single-bus power system, when non-linearities and detailed power components models come into play the time-independency of the stability for such share may not be valid. This confirmed the need for the inclusion of a recommendation on the EV response time (Recommendation 2). Results show that for EV 
response times of $1 \mathrm{~s}$ and $4 \mathrm{~s}$ the stability was assured, whereas for $7 \mathrm{~s}$ (very close to the calculated $T_{\text {limit }}=8$ s), slowly damped oscillations appeared before settling to the steady-state frequency value. On the one hand, this proved the need for the simultaneous fulfilment of the two proposed requirements when including EV fleets as primary reserve providers. On the other hand, these results confirmed that the proposed recommendations can be a valuable tool for defining benchmark limit values to be implemented for subsequent sets of simulation studies in exhaustively modelled power systems.

As a discussion topic, the authors would like to point out that in case relatively large EV share and too slow EV responses, the power system instability conditions may be prevented only if additional counteractions are taken. An example could be the inclusion of a rate limiter able to smooth the aggregated EV response. When implementing a rate limiter the system frequency could be recovered in a safe band around the steady-state value and uncontrolled growing oscillations could be prevented. Nonetheless, the nadir and the settling time would be dramatically influenced by the sensitiveness of the employed limiter, and new slowly damped oscillations around the steady-state frequency may appear. Rate limiters should then be properly tuned and all the eventual introduced effects should be taken into account.

To conclude, the proposed recommendations should be considered as a tool for power system studies to be utilized as a benchmark for further grid analysis in more complete and complex simulation environments. In fact, this was done in the second part of this paper with the detailed implementation of the Bornholm Island power system. However, it is worth mentioning that possible additional precautions could be deployed and included with the aim at assuring safe and reliable operation also for larger EV shares and/or delays. In fact, the authors recognize that in some cases a smooth overall response could be needed, achievable for instance by introducing additional requirements on the whole aggregated EV fleet response. These aspects are being investigated within future works.

\section{Acknowledgements}

The authors would like to thank the financial support of the Danish EUDP program for the project 'ACES - Across Continent Electric Vehicle Services'. (Grant EUDP17-I-12499, website: www.aces-bornholm.eu).' 


\section{References}

[1] E. L. Karfopoulos, K. A. Panourgias, and N. D. Hatziargyriou, "Distributed Coordination of Electric Vehicles providing V2G Regulation Services," IEEE Transactions on Power Systems, vol. 31, no. 4, pp. 2834-2846, 2016.

[2] S. Izadkhast, P. Garcia-Gonzalez, and P. Frías, "An Aggregate Model of Plug-In Electric Vehicles for Primary Frequency Control," IEEE Transactions on Power Systems, vol. 30, no. 3, pp. 1475-1482, 2015.

[3] C. Ziras, J. Hu, S. You, and H. W. Bindner, "Modelling the aggregated dynamic response of electric vehicles," in 2017 IEEE PES Innovative Smart Grid Technologies Conference Europe (ISGT-Europe), 2017, pp. 1-6.

[4] E. Sortomme and K. W. Cheung, "Intelligent Dispatch of Electric Vehicles Performing Vehicle-to-Grid Regulation," in 2012 IEEE International Electric Vehicle Conference, 2012, pp. 1-6.

[5] C. Peng, J. Zou, and L. Lian, "Dispatching strategies of electric vehicles participating in frequency regulation on power grid: A review," Renewable and Sustainable Energy Reviews, vol. 68, no. July 2015, pp. 147-152, 2017.

[6] M. González Vayá and G. Andersson, "Combined Smart-Charging and Frequency Regulation for Fleets of Plug-in Electric Vehicles," in 2013 IEEE Power \& Energy Society General Meeting, 2013, pp. 1-5.

[7] K. Clement-Nyns, E. Haesen, and J. Driesen, "The impact of vehicle-to-grid on the distribution grid," Electric Power Systems Research, vol. 81, no. 1, pp. 185-192, 2011.

[8] H. R. Galiveeti, A. K. Goswami, and N. B. Dev Choudhury, "Impact of plug-in electric vehicles and distributed generation on reliability of distribution systems," Engineering Science and Technology, an International Journal, vol. 21, no. 1 , pp. 50-59, 2018.

[9] Y. Kongjeen and K. Bhumkittipich, "Impact of Plug-in Electric Vehicles Integrated into Power Distribution System Based on Voltage-Dependent Power Flow Analysis," Energies, vol. 11, no. 6, p. 1571, 2018.

[10] J. C. Hernández, F. Sanchez-Sutil, P. G. Vidal, and C. Rus-Casas, "Primary frequency control and dynamic grid support for vehicle-to-grid in transmission systems," International Journal of Electrical Power and Energy Systems, vol. 100, no. July 2017, pp. 152-166, 2018.

[11] P. M. R. Almeida, F. J. Soares, and J. A. P. Lopes, "Electric vehicles contribution for frequency control with inertial emulation," Electric Power Systems Research, vol. 127, pp. 141-150, 2015.

[12] J. Meng, Y. Mu, H. Jia, J. Wu, X. Yu, and B. Qu, "Dynamic frequency response from electric vehicles considering travelling behavior in the Great Britain power system," Applied Energy, vol. 162, pp. 966-979, 2016.

[13] J. A. P. Lopes, P. M. R. Almeida, F. J. Soares, and C. L. Moreira, "Electric vehicles in isolated power systems: Conceptual framework and contributions to improve the grid resilience," IFAC Proceedings Volumes, vol. 1, no. PART 1, pp. 24-29, 2010.

[14] M. Vahedipour-Dahraie, H. Rashidizaheh-Kermani, H. Najafi, A. Anvari-Moghaddam, and J. Guerrero, "Coordination of EVs Participation for Load Frequency Control in Isolated Microgrids," Applied Sciences, vol. 7, no. 6, p. 539, 2017.

[15] K. Knezović, M. Marinelli, A. Zecchino, P. B. Andersen, and C. Træholt, "Supporting involvement of electric vehicles in distribution grids: Lowering the barriers for a proactive integration," Energy, 2017.

[16] C. Ziras, A. Zecchino, and M. Marinelli, "Response Accuracy and Tracking Errors with Decentralized Control of Commercial V2G Chargers," in 20th Power Systems Computation Conference (PSCC 2018), 2018, pp. 1-7.

[17] J. A. Short, D. G. Infield, and L. L. Freris, "Stabilization of grid frequency through dynamic demand control," IEEE Transactions on Power Systems, vol. 22, no. 3, pp. 1284-1293, 2007.

[18] C. Ziras, E. Vrettos, and S. You, "Controllability and stability of primary frequency control from thermostatic loads with delays," Journal of Modern Power Systems and Clean Energy, vol. 5, no. 1, pp. 43-54, 2017.

[19] M. R. Vedady Moghadam, R. T. B. Ma, and R. Zhang, "Distributed Frequency Control in Smart Grids via Randomized Demand Response," IEEE Transactions on Smart Grid, vol. 5, no. 6, pp. 2798-2809, 2014.

[20] M. R. V. Moghadam, R. Zhang, and R. T. B. Ma, "Distributed Frequency Control via Randomized Response of Electric Vehicles in Power Grid," IEEE Transactions on Sustainable Energy, vol. 7, no. 1, pp. 312-324, 2016.

[21] S. Izadkhast, S. Member, P. Garcia-gonzalez, and P. Fr, "Design of Plug-In Electric Vehicle' s Frequency-Droop Controller for Primary Frequency Control and Performance Assessment," vol. 32, no. 6, pp. 4241-4254, 2017.

[22] M. Rezkalla, A. Zecchino, S. Martinenas, A. M. Prostejovky, and M. Marinelli, "Comparison between Synthetic Inertia and Fast Frequency Containment Control on Single Phase EVs in a Microgrid," Applied Energy, 2017.

[23] M. Marinelli, S. Martinenas, K. Knezović, and P. B. Andersen, "Validating a centralized approach to primary frequency control with series-produced electric vehicles," Journal of Energy Storage, vol. 7, pp. 63-73, 2016.

[24] "Nissan, enel and nuvve operate world's first fully commercial vehicle-to-grid hub in denmark," 2016. [Online]. Available: http://www.nissan-helsingor.dk/index.php/om-os/nyheder/show/news/id/4. [Accessed: 19-Oct-2018].

[25] "The Parker project," http://parker-project.com, 2016. .

[26] "ACES project - across continents electric vehicle services," http://aces-bornholm.eu, 2017. .

[27] A. Zecchino, A. Thingvad, P. B. Andersen, and M. Marinelli, "Suitability of Commercial V2G CHAdeMO Chargers 
for Grid Services," in EVS 31 \& EVTeC 2018, 2018, pp. 1-7.

[28] ENTSO-E, "ENTSO-E, Continental Europe Operation Handbook," 2009.

[29] Energinet.dk, "Danish Technical Standard: ancillary services to be delivered in Denmark - tender conditions," https://en.energinet.dk/-/media/Energinet/El-RGD/Dokumenter/Ancillary-services-to-be-delivered-in-Denmark.pdf, 2017. .

[30] J. Østergaard and J. Nielsen, "The Bornholm Power System An Overview," 2008.

[31] Y. Chen, Z. Xu, and J. Østergaard, "Frequency Analysis for Planned Islanding Operation in the Danish Distribution System - Bornholm," Proceedings of the Universities Power Engineering Conference, pp. 9-13, 2008.

[32] E. James-Smith and M. Togeby, "Security of Supply of Bornholm - Demand Side Options for System Reserves," 2007.

[33] IEA International Energy Agency, "Nordic EV Outlook 2018," 2018.

[34] P. Kundur, Power system stability and control. 1994.

[35] S. R. P. Malik and G. S. Hope, "An adaptive control scheme for speed control of diesel driven power-plants," IEEE Transactions on Energy Conversion, vol. 6, no. 4, pp. 605-611, 1991.

[36] K. Gu, J. Chen, and V. Kharitonov, Stability of Time- Delay Systems. Secaucus, NJ, USA: Birkhauser Boston, Inc., 2003.

[37] C. Hjalmar, "Danish National Travel Survey," 2017. 